Ext reme ul travi ol et spect roscopy and at om c model s of hi ghl y charged heavy i ons i $n$ the Lar ge Hel i cal Devi ce

\begin{tabular}{|l|l|}
\hline $\begin{array}{l}\text { j our nal or } \\
\text { publ i cat i on ti t l e }\end{array}$ & Pl asma Physi cs and Cont rol I ed Fusi on \\
\hline vol ume & 59 \\
\hline page range & 014009 \\
\hline year & $2017-10$ - 18 \\
\hline URL & ht t p: //hdl . handl e. net /10655/00012518 \\
\hline
\end{tabular}




\title{
Extreme ultraviolet spectroscopy and atomic models of highly charged heavy ions in the Large Helical Device
}

\author{
C Suzuki ${ }^{1}$, I Murakami ${ }^{1,2}$, F Koike ${ }^{3}$, N Tamura ${ }^{1}$, H A Sakaue ${ }^{1}$, \\ S Morita ${ }^{1,2}$, M Goto ${ }^{1,2}$, D Kato ${ }^{1,2}$, H Ohashi $^{4}$, T Higashiguchi ${ }^{5}$, \\ S Sudo ${ }^{6}$, G O'Sullivan \\ 1 National Institute for Fusion Science, National Institutes of Natural Sciences, 322-6 \\ Oroshi-cho, Toki 509-5292, Japan \\ 2 SOKENDAI (The Graduate University for Advanced Studies), Shonan Village, \\ Hayama 240-0193, Japan \\ 3 Sophia University, 7-1 Kioi-cho, Chiyoda-ku, Tokyo 102-8554, Japan \\ ${ }^{4}$ University of Toyama, 3190 Gofuku, Toyama 930-8555, Japan \\ ${ }^{5}$ Utsunomiya University, 7-1-2 Yoto, Utsunomiya 321-8585, Japan \\ ${ }^{6}$ Chubu University, 1200 Matsumoto-cho, Kasugai 487-8501, Japan \\ 7 University College Dublin, Belfield, Dublin 4, Ireland \\ E-mail: csuzuki@nifs.ac.jp
}

\begin{abstract}
We report recent results of extreme ultraviolet (EUV) spectroscopy of highly charged heavy ions in plasmas produced in the Large Helical Device (LHD). The LHD is an ideal source of experimental databases of EUV spectra because of high brightness and low opacity, combined with the availability of pellet injection systems and reliable diagnostic tools. The measured heavy elements include tungsten, tin, lanthanides and bismuth, which are motivated by ITER as well as a variety of plasma applications such as EUV lithography and biological microscopy. The observed spectral features drastically change between quasicontinuum and discrete depending on the plasma temperature, which leads to some new experimental identifications of spectral lines. We have developed collisional-radiative models for some of these ions based on the measurements. The atomic number dependence of the spectral feature is also discussed.
\end{abstract}

PACS numbers: 32.30.Jc, 32.70.-n, 52.25.Os

Keywords: EUV spectroscopy, highly charged ions, heavy elements, LHD, UTA

Submitted to: Plasma Phys. Control. Fusion 


\section{Introduction}

Among various types of plasma sources, magnetically confined torus plasmas for fusion research have some unique characteristics: high emissivity, low opacity and high temperature of the order of $\mathrm{keV}$. In addition, a number of diagnostic tools with high spatial and temporal resolutions are usually prepared for the measurements of plasma parameters in these facilities. Therefore, some tokamaks have been exploited as light sources for basic spectroscopic studies of highly charged ions by introducing impurities into high temperature plasmas $[1,2,3]$.

The Large Helical Device (LHD) at the National Institute for Fusion Science is a large scale facility for fusion research equipped with superconducting helical coils and neutral beam injection (NBI) systems [4]. The LHD is considered to be more appropriate for the above mentioned purpose because of higher flexibility of plasma density and temperature, allowance for larger amounts of impurities and plasma stability. Temporal evolution of electron temperature and density profiles is precisely measured by an existing Thomson scattering diagnostic system with high spatial and temporal resolution $[5,6]$. Multiple pellet injection systems $[7,8]$ and extreme ultraviolet (EUV) spectrometers $[9,10]$ are routinely running for studies on impurity transport. Accordingly, we have extensively promoted so far in the LHD a variety of spectroscopic studies relevant not only to fusion research but also to astrophysics, plasma applications and basic atomic physics $[11,12,13,14,15]$.

Figure 1 shows the 5 th and 6 th period elements which have been injected into LHD plasmas to date. Among them, tungsten (W) is the most frequently injected element as it is a planned divertor material in ITER $[11,16,17,18]$. Some of the other elements listed here are related to industrial applications to short-wavelength light sources. For example, tin $(\mathrm{Sn})$ and gadolinium $(\mathrm{Gd})$ are being considered as candidate materials for the next generation light sources for EUV lithography [19, 20, 21, 22]. Bismuth (Bi) is a candidate material for a tabletop light source in the water window range $(2.3-4.4 \mathrm{~nm})$ for high-contrast biological microscopy [23]. We have also studied a number of elements adjacent to them in the periodic table to investigate atomic number $(Z)$ dependence in terms of basic atomic physics [15, 24, 25, 26, 27].

This paper reports recent results of EUV spectroscopy of highly charged heavy ions

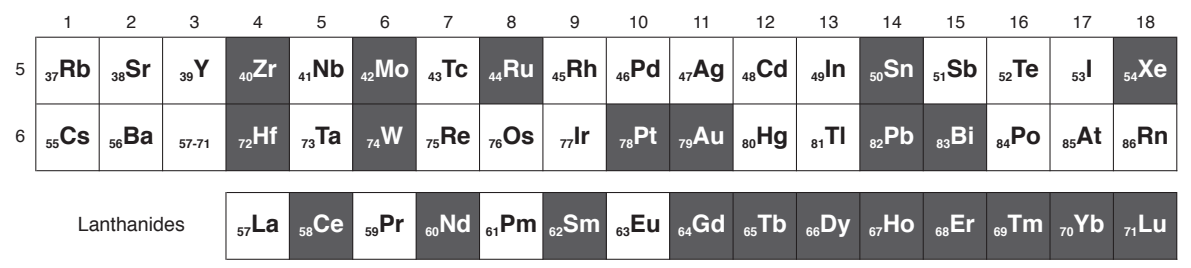

Figure 1. A partial periodic table of the elements for the 5 th and 6 th periods. Symbols of the elements which have been injected into LHD plasmas to date are displayed in white on dark. 


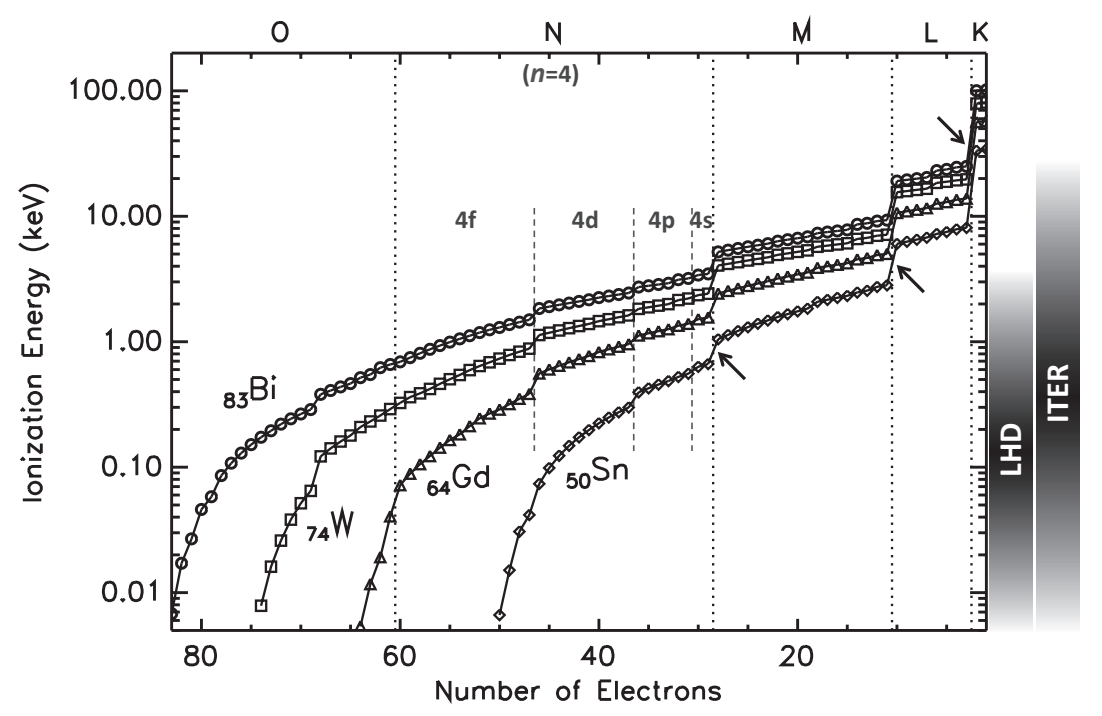

Figure 2. Ionization energies of tin (diamonds), gadolinium (triangles), tungsten (squares) and bismuth (circles) ions as functions of number of electrons. Energy ranges corresponding to the temperature ranges in LHD and ITER plasmas are roughly displayed on the right by grey scales.

in LHD plasmas. Some of the background necessary to interpret the spectra is reviewed in section 2 with an emphasis on unresolved transition arrays (UTA). The experimental procedure to obtain temperature dependent spectra is briefly described in section 3 , and a typical example of the result is shown in section 4 . The $Z$ dependence of the EUV spectra is discussed in section 5 together with the identification of some isolated spectral lines. Recent developments of the collisional-radiative (CR) models compared with the experiments are shown in section 6 followed by a brief summary in section 7 .

\section{Unresolved transition array}

In the EUV wavelength region (roughly 1-15 nm), the emission from highly charged heavy ions often appears as an unresolved transition array (UTA) [28], a quasicontinuum band-like spectrum composed of millions of unresolvable lines. The general spectral features of the heavy ions relevant to this study are briefly described in this section for easier understanding of the discussions in the following sections. Ionization energies of $\mathrm{Sn}, \mathrm{Gd}, \mathrm{W}$ and $\mathrm{Bi}$ ions are plotted in figure 2 as functions of the number of electrons. The corresponding outermost electron shells are denoted on the top of figure 2 . It is clearly seen that the ionization energy jumps at the boundaries where the outermost shell changes, as indicated by the arrows in figure 2. As shown by a grey scale on the right of figure 2, the electron temperature in LHD is reduced to be lower than $2-3 \mathrm{keV}$ when a sufficient amount of heavy element is introduced. Therefore, heavy ions having outermost $N(n=4)$ shell electrons, the ionization energies of which are roughly 0.1$3 \mathrm{keV}$, would be the dominant emitters in LHD plasmas. Photons corresponding to 

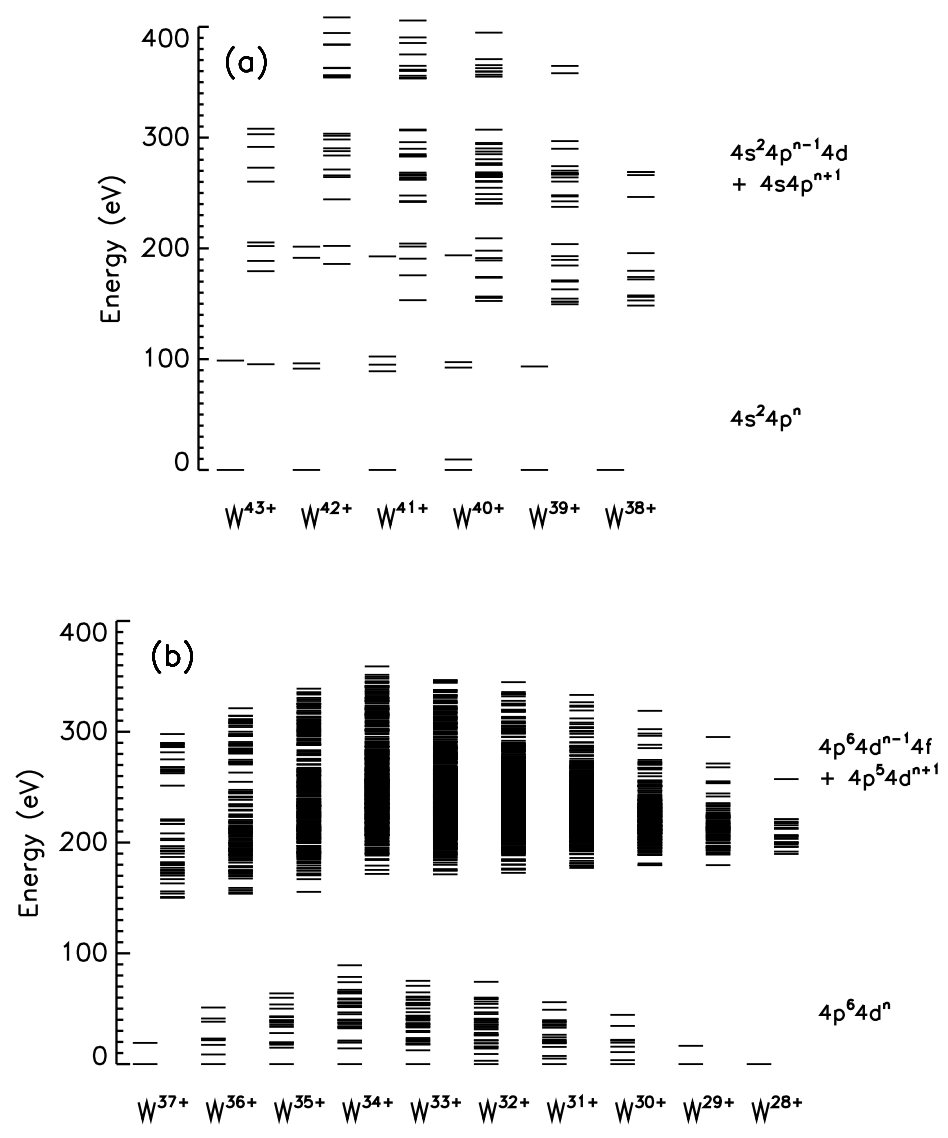

Figure 3. Calculated energy level diagrams of tungsten ions having outermost (a) 4p and (b) $4 \mathrm{~d}$ subshell electrons in their ground states. The levels for the ground and the first excited configurations are drawn on the left and right sides for each ion. The first excited configurations are the mixtures of the type (a) $4 \mathrm{~s}^{2} 4 \mathrm{p}^{n-1} 4 \mathrm{~d}+4 \mathrm{~s} 4 \mathrm{p}^{n+1}$ and (b) $4 \mathrm{p}^{6} 4 \mathrm{~d}^{n-1} 4 \mathrm{f}+4 \mathrm{p}^{5} 4 \mathrm{~d}^{n+1}$.
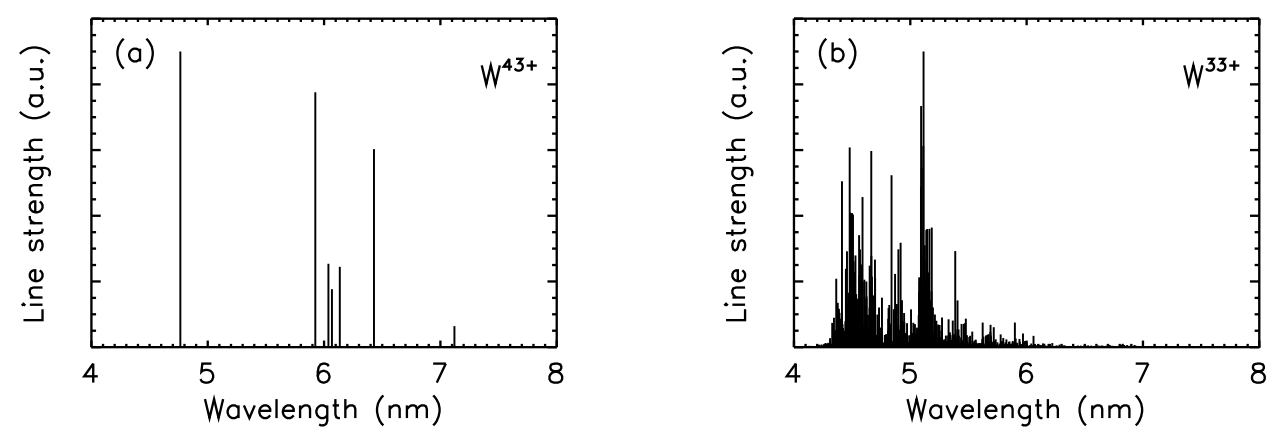

Figure 4. Calculated spectra of the normalized line strengths of (a) Ga-like $\mathrm{W}^{43+}$ and (b) Nb-like $\mathrm{W}^{33+}$ arising from the energy levels shown in figure 3. 
this energy range are mainly in the EUV region. As shown in figure 2, these ions are subdivided by the outermost subshells: 4s, 4p, 4d and 4f. Small jumps in the ionization energies are seen at the boundaries between the adjacent subshells.

The number of energy levels largely depends on the number of electrons in the outermost subshells. For example, energy level diagrams of tungsten ions having outermost $4 \mathrm{p}$ and $4 \mathrm{~d}$ subshells are illustrated in figure 3 , and the corresponding spectra of the normalized line strengths ( $\mathrm{g} A$ values) of the $n=4-4(\Delta n=0)$ transitions are drawn in figure 4 for Ga-like $\mathrm{W}^{43+}$ (single electron $4 \mathrm{p}$ subshell) and Nb-like $\mathrm{W}^{33+}$ (half filled $4 \mathrm{~d}$ subshell) ions. The atomic structures and line strengths were calculated with the Flexible Atomic Code (FAC) [29] including the minimum numbers of configurations and their mixings: i.e., $4 \mathrm{~d}^{6} 4 \mathrm{~d}^{n}$ and the mixed configuration of the type $4 \mathrm{p}^{6} 4 \mathrm{~d}^{n-1}+4 \mathrm{p}^{5} 4 \mathrm{~d}^{n+1}$ for ions with outermost $4 \mathrm{~d}$ subshell. It can be easily understood from figure 4 that the ions with outermost $4 \mathrm{p}$ and $4 \mathrm{~d}$ subshells tend to yield discrete and quasicontinuum UTA spectral features, respectively. Also, figure 3 (b) indicates that the average $n=4-$ 4 transition energy does not change so much with ion charge, which gives rise to the intense band of emission contributed from a number of charge states. On the other hand, $n=4-5$ or $4-6(\Delta n \neq 0)$ transitions tend to form a series of UTAs separated by charge states in the higher energy (shorter wavelength) region. This phenomenon has been demonstrated experimentally in the LHD and laser-produced plasmas for tungsten ions $[18,30]$.

\section{Experimental}

Since the experimental procedure in the LHD has already been described in our earlier publications [15, 25, 26], a brief explanation is given here with an emphasis on the heating scheme to obtain a variety of electron temperatures. Typical examples of the temporal evolution of various parameters in a discharge with a thulium (Tm) pellet injection are shown in figure 5. The electron temperature profiles measured with the Thomson scattering diagnostic $[5,6]$ at 5.0, 5.5 and $6.0 \mathrm{~s}$ in the same discharge are shown in figure 6. Among five NBI beamlines available, three beams (NBI \#1-3) are injected tangentially with an acceleration voltage of $180 \mathrm{keV}$, while the other two (NBI \#4-5) are injected perpendicularly with an acceleration voltage of $40 \mathrm{keV}$ [31]. This means that the tangential and perpendicular beams contribute primarily to electron and ion heating, respectively [31]. A small amount $\left(\simeq 10^{17}\right.$ atoms) of Tm was injected at $3.8 \mathrm{~s}$ with a tracer encapsulated solid pellet (TESPEL) [7] while the tangential beams were injected during 3.3-5.3 s to sustain a high electron temperature above $2 \mathrm{keV}$. In order to decrease the electron temperature effectively, the tangential beams were switched to the vertical beams at $5.3 \mathrm{~s}$. As a result, the core electron temperature finally dropped to zero at around $5.6 \mathrm{~s}$ because of the formation of a hollow temperature profile as shown in figure 6 (c).

Temporal evolutions of the EUV spectra are routinely recorded with two types of grazing incidence spectrometers $[9,10]$. In this article, we mainly describe the spectra 


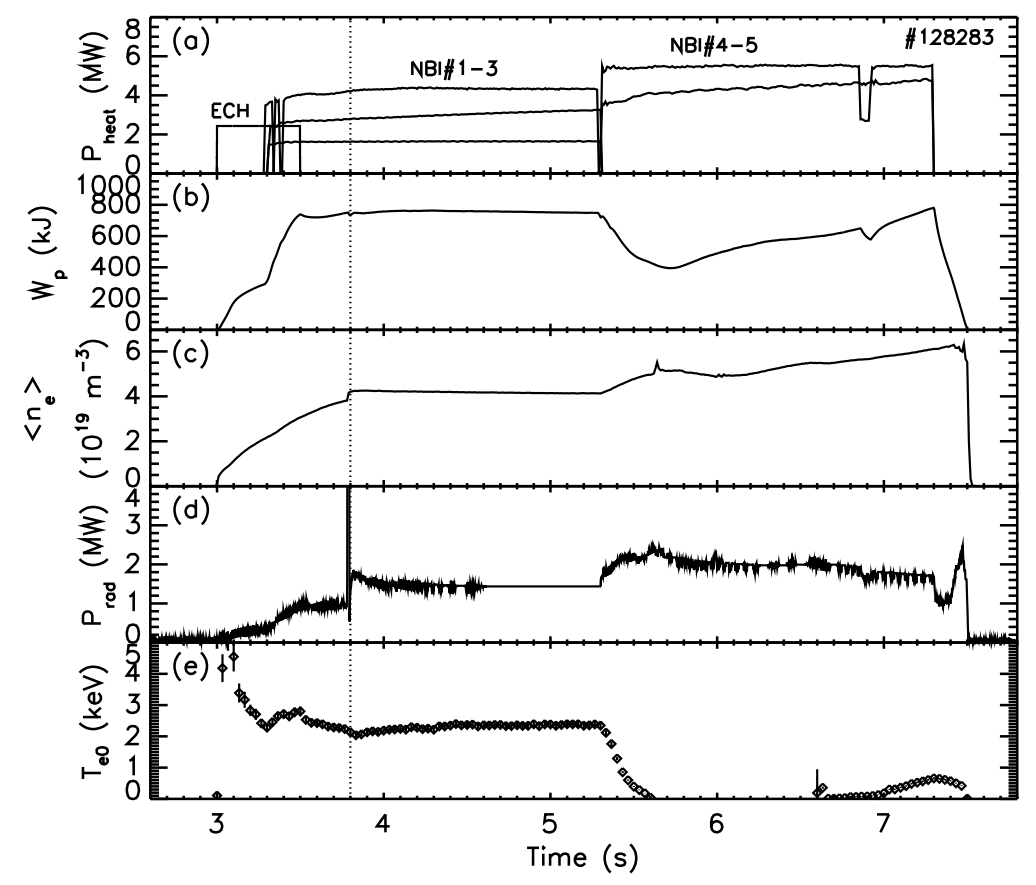

Figure 5. Temporal evolution of (a) heating power $\left(P_{\text {heat }}\right)$, (b) plasma stored energy $\left(W_{\mathrm{p}}\right),(\mathrm{c})$ line averaged electron density $\left(\left\langle n_{\mathrm{e}}\right\rangle\right),(\mathrm{d})$ total radiated power $\left(P_{\mathrm{rad}}\right)$ and $(\mathrm{e})$ central electron temperature $\left(T_{\mathrm{e} 0}\right)$ in an LHD discharge with a Tm pellet injected at $3.8 \mathrm{~s}$.

measured by a $2 \mathrm{~m}$ Schwob-Fraenkel spectrometer [9] every 0.1 or $0.2 \mathrm{~s}$. The spectral resolution is around $0.01 \mathrm{~nm}$ with a grating of 600 grooves $/ \mathrm{mm}$. The absolute wavelength is calibrated using the positions of known lines of intrinsic and intentionally injected impurity ions, which results in an uncertainty of about $\pm 0.005 \mathrm{~nm}$.

\section{Temperature dependence of EUV spectra}

The discussions in section 2 imply that the spectral feature would change between discrete and quasicontinuum UTA according to whether the outermost subshell of the dominant ion stages is $4 \mathrm{p}$ or $4 \mathrm{~d}$. For example, temperature dependence of the spectra around $6.5 \mathrm{~nm}$ from dysprosium (Dy) ions in an LHD plasma is shown in figure 7 . The maximum electron temperature $\left(T_{\mathrm{e}_{-} \max }\right)$ along the line of sight for each timing is shown in each panel. When $T_{\text {e_max }}$ was $1.7 \mathrm{keV}$, a discrete spectrum with less UTA feature was observed as shown in the top panel. These isolated lines mainly originate from $n=4-4$ transitions of Cu-, Zn- and Ga-like ions having $4 \mathrm{~s}, 4 \mathrm{~s}^{2}$ and $4 \mathrm{~s}^{2} 4 \mathrm{p}$ ground configurations. Indeed, the lines due to the $3 \mathrm{~d}^{10} 4 \mathrm{p}^{2} \mathrm{P}_{1 / 2}-3 \mathrm{~d}^{10} 4 \mathrm{~d}^{2} \mathrm{D}_{3 / 2}$ and $3 \mathrm{~d}^{10} 4 \mathrm{~d}^{2} \mathrm{D}_{3 / 2}-3 \mathrm{~d}^{10} 4 \mathrm{f}^{2} \mathrm{~F}_{5 / 2}$ transitions of $\mathrm{Cu}$-like ions are easily found at $6.902 \mathrm{~nm}$ and $8.546 \mathrm{~nm}$, respectively [26]. When $T_{\text {e_max }}$ decreased to $1.2 \mathrm{keV}$, a UTA feature with a maximum at around $6.5 \mathrm{~nm}$ developed with a bandwidth of about $0.5 \mathrm{~nm}$. As the temperature decreases, the peak 


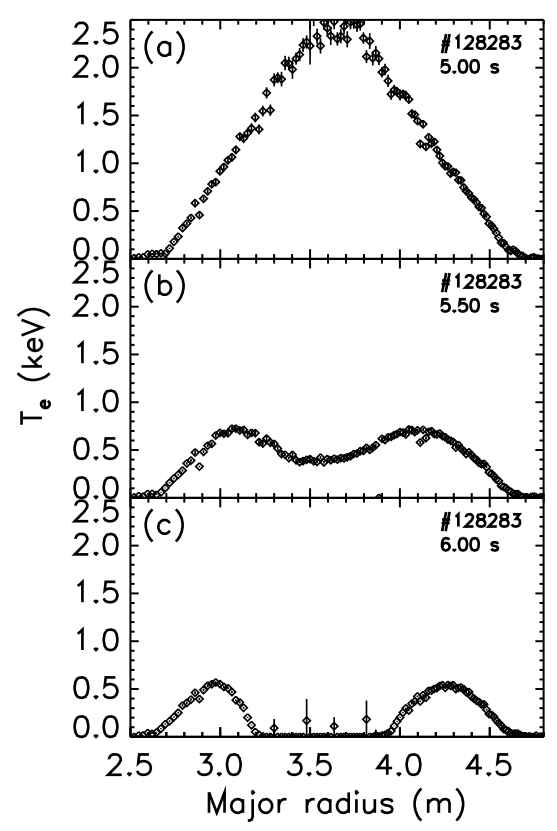

Figure 6. Electron temperature profiles at (a) $5.0 \mathrm{~s}$, (b) $5.5 \mathrm{~s}$ and (c) $6.0 \mathrm{~s}$ in the discharge shown in figure 5 .

position of the UTA moves to shorter wavelength and the bandwidth becomes narrower. Finally, a very narrowed UTA feature was formed with a maximum at $6.35 \mathrm{~nm}$ at $T_{\mathrm{e} \_ \text {max }}=0.4 \mathrm{keV}$ as shown in the last panel. The dominant ion stages are considered to be Pd- and Ag-like ions because an isolated double peak around $6.6 \mathrm{~nm}$ is identified as the $4 \mathrm{~d}^{10} 4 \mathrm{f}^{2} \mathrm{~F}-4 \mathrm{~d}^{9} 4 \mathrm{f}^{2}{ }^{2} \mathrm{G}$ transition of Ag-like Dy ${ }^{19+}$ ions, and the highest peak at $6.280 \mathrm{~nm}$ is close to the previously reported value of the $4 \mathrm{~d}^{10}{ }^{1} \mathrm{~S}_{0}-4 \mathrm{~d}^{9} 4 \mathrm{f}^{1} \mathrm{P}_{1}$ transition of Pd-like $\mathrm{Dy}^{20+}$ ions [26].

It seems curious that the UTA peak moves slightly to longer wavelength with increasing temperature because electrons in higher charge states generally feel a larger potential well from nuclear charge. This behaviour of the UTA wavelength peculiar to $n=4-4(\Delta n=0)$ transition is mainly due to the effect of multi-electron correlation energy among $n=4$ electrons $[32,33]$, which can be qualitatively reproduced by the FAC code calculation shown in figure 8. In contrast, the UTA positions of the $\Delta n \neq 0$ transitions move to shorter wavelength with increasing charge, which results in a series of UTAs separated by charge state as mentioned in section 2 .

\section{Z dependence of EUV spectra}

An empirical quasi-Moseley's law has recently been derived for the peak wavelength of the $n=4-4$ UTA emission, $\lambda_{\mathrm{UTA}}$, from highly charged ions with $Z$ from 50 onward based on the spectra observed in Nd:YAG laser-produced plasmas (LPP) [34], which gives

$$
\lambda_{\mathrm{UTA}}=a R_{\infty}^{-1}(Z-s)^{-b},
$$




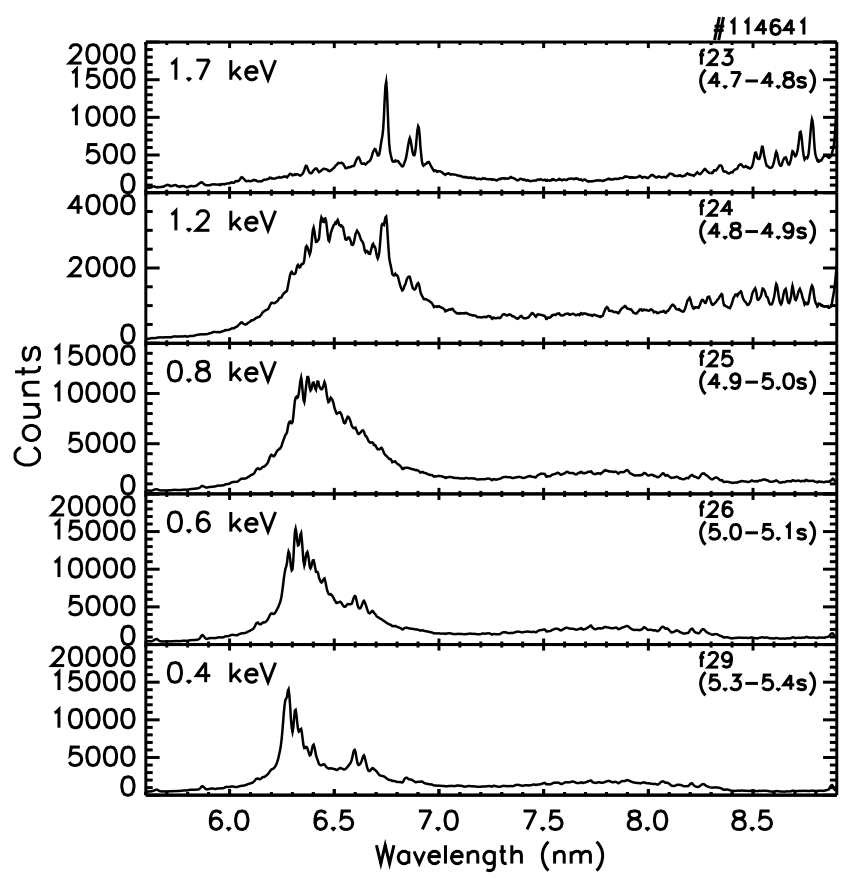

Figure 7. Temperature dependence of the measured EUV spectra from Dy ions around $6.5 \mathrm{~nm}$. The maximum electron temperatures along the line of sight are displayed in each panel.

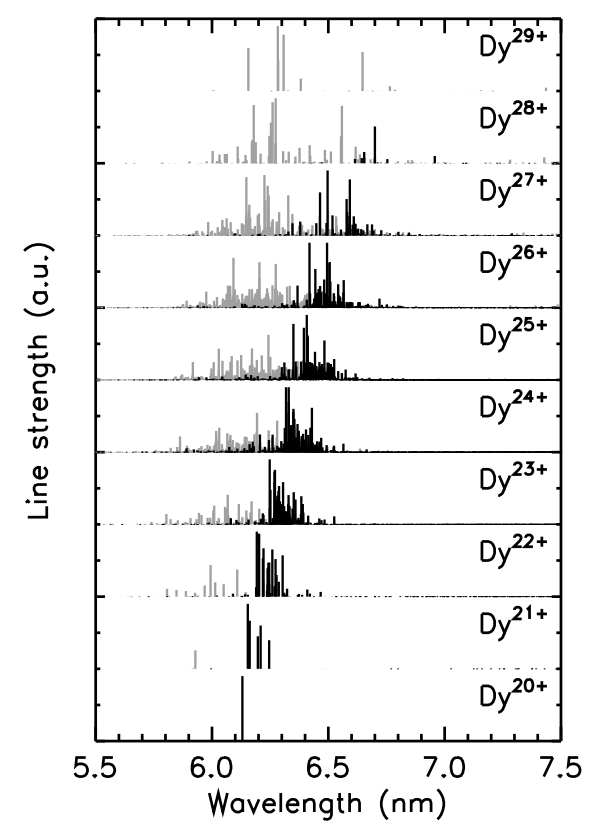

Figure 8. Calculated spectra of the normalized line strengths of Dy ions having outermost $4 \mathrm{~d}$ electrons. Transitions relevant to $4 \mathrm{p}^{6} 4 \mathrm{~d}^{n-1} 4 \mathrm{f}$ and $4 \mathrm{p}^{5} 4 \mathrm{~d}^{n+1}$ configurations are drawn by black and grey lines, respectively. 


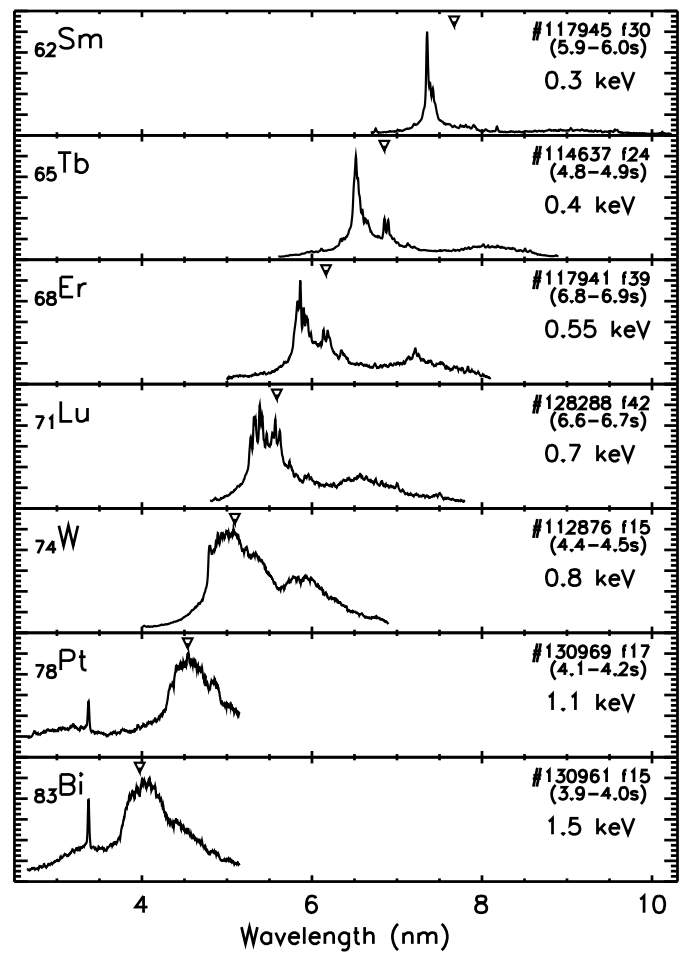

Figure 9. $Z$ dependence of the normalized EUV spectra from heavy ions with $Z$ ranging from 62 to 83 . The time frames were selected so that the maximum electron temperature along the line of sight is roughly equal to the ionization energies of Ag-like ions. The UTA peak positions predicted from the quasi-Moseley's law [34] are marked by the triangles in each panel.

Table 1. List of the isolated lines identified experimentally for the first time in the LHD. The uncertainty of the wavelengths measured in the LHD is estimated to be less than $\pm 0.005 \mathrm{~nm}$. The details of the lines from Ho and Tm ions will be reported soon in a separate paper.

\begin{tabular}{|c|c|c|c|}
\hline Ion & Transition & Wavelength (nm) & Ref. \\
\hline \multicolumn{4}{|l|}{ Ni-like } \\
\hline $\mathrm{Nd}^{32+}$ & $3 \mathrm{~d}_{3 / 2} 4 \mathrm{p}_{1 / 2}-3 \mathrm{~d}_{3 / 2} 4 \mathrm{~d}_{3 / 2}$ & 7.413 & [25] \\
\hline \multicolumn{4}{|l|}{$\mathrm{Cu}$-like } \\
\hline $\mathrm{Tb}^{36+}$ & $3 \mathrm{~d}^{10} 4 \mathrm{p}{ }^{2} \mathrm{P}_{1 / 2}-3 \mathrm{~d}^{10} 4 \mathrm{~d}^{2} \mathrm{D}_{3 / 2}$ & 7.203 & [26] \\
\hline $\mathrm{Tb}^{36+}$ & $3 \mathrm{~d}^{10} 4 \mathrm{~d}^{2} \mathrm{D}_{3 / 2}-3 \mathrm{~d}^{10} 4 \mathrm{f}^{2} \mathrm{~F}_{5 / 2}$ & 8.796 & [26] \\
\hline $\mathrm{Ho}^{38+}$ & $3 \mathrm{~d}^{10} 4 \mathrm{p}^{2} \mathrm{P}_{1 / 2}-3 \mathrm{~d}^{10} 4 \mathrm{~d}^{2} \mathrm{D}_{3 / 2}$ & 6.614 & \\
\hline $\operatorname{Tm}^{40+}$ & $3 \mathrm{~d}^{10} 4 \mathrm{p}^{2} \mathrm{P}_{1 / 2}-3 \mathrm{~d}^{10} 4 \mathrm{~d}^{2} \mathrm{D}_{3 / 2}$ & 6.072 & \\
\hline \multicolumn{4}{|l|}{ Ag-like } \\
\hline $\operatorname{Tm}^{22+}$ & $4 \mathrm{~d}^{10} 4 \mathrm{f}^{2} \mathrm{~F}_{7 / 2}-4 \mathrm{~d}^{9} 4 \mathrm{f}^{2}{ }^{2} \mathrm{G}_{9 / 2}$ & 5.933 & \\
\hline $\operatorname{Tm}^{22+}$ & $4 \mathrm{~d}^{10} 4 \mathrm{f}^{2} \mathrm{~F}_{5 / 2}-4 \mathrm{~d}^{9} 4 \mathrm{f}^{2}{ }^{2} \mathrm{G}_{7 / 2}$ & 5.986 & \\
\hline
\end{tabular}


where $a=21.86 \pm 12.09, b=1.52 \pm 0.12, s=23.23 \pm 2.87$ and $R_{\infty}$ is the Rydberg constant. This empirical law is similar to the classical Moseley's law in which $a=4 / 3, b=2$ and $s=1$ for $K_{\alpha}$ lines of characteristic X-rays.

Figure 9 shows the $Z$ dependence of the normalized EUV spectra from some of the heavy ions with $Z$ ranging from 62 to 83 measured in the LHD, together with the UTA peak positions predicted from (1) denoted by triangles. The time frames were chosen at the timings when $T_{\text {e_max }}$ roughly agree with the corresponding ionization energies of Ag-like ions so as to follow the emission from open $N$ shell ions having similar electron configurations. The LHD spectra roughly follows (1) for higher $Z$ elements, while the peak position slightly shifts to shorter wavelength from the prediction for lower $Z$ elements. This is probably because of the difference between charge state distributions in LHD plasmas and Nd:YAG LPPs. Because of higher opacity and lower temperature in the YAG LPPs, the spectra from the LPPs tend to be broader and include emissions from lower charge states having 4 f outermost subshell, which appear on the longer wavelength side [34].

As a result of the survey of the EUV spectra from a wide range of $Z$, we have identified some of the isolated spectral lines from ions with relatively simple electron configurations such as $\mathrm{Ni}$-, $\mathrm{Cu}$ - and $\mathrm{Ag}$-like ions. The lines of neodymium (Nd), terbium $(\mathrm{Tb})$, holmium ( $\mathrm{Ho}$ ) and $\mathrm{Tm}$ ions identified experimentally for the first time in the LHD are listed in table $1[25,26]$. The $\mathrm{Ni}$ - and $\mathrm{Cu}$-like lines were observed under high temperature conditions above $1.5 \mathrm{keV}$, while the $\mathrm{Ag}$-like ions were observed under low temperature conditions below $0.6 \mathrm{keV}$.

\section{Modelling}

For tungsten ions, we have constructed a collisional-radiative (CR) model in which rate equations for the population density $n_{\mathrm{q}}(i)$ of an excited level $i$ for $q$ th ion stage is given by

$$
\begin{aligned}
\frac{d n_{q}(i)}{d t}= & \sum_{k<i} C_{q}(k, i) n_{\mathrm{e}} n_{q}(k)+\sum_{j>i}\left\{C_{q}(j, i) n_{\mathrm{e}}+A_{q}(j, i)\right\} n_{q}(j) \\
& -\sum_{k<i}\left\{C_{q}(i, k) n_{\mathrm{e}} n_{q}(i)+A_{q}(i, k)\right\} n_{q}(i)-\sum_{j>i} C_{q}(i, j) n_{\mathrm{e}} n_{q}(i) \\
& -S_{q}(i) n_{\mathrm{e}} n_{q}(i),
\end{aligned}
$$

where $C_{q}(i, j)$ is the electron impact excitation or de-excitation rate coefficient between levels $i$ and $j, n_{\mathrm{e}}$ is the electron density, $A_{q}(i, j)$ is the spontaneous transition probability between levels $i$ and $j$ and $S_{q}(i)$ is the electron impact ionization rate coefficient of level $i$. The atomic level structure, $C_{q}(i, j), A_{q}(i, j)$ and $S_{q}(i)$ are calculated with the HULLAC code, a fully relativistic code based on the Dirac Hamiltonian [35]. Recombination processes are not included in the model because of the difficulties in the calculations of dielectronic recombination rate coefficients systematically for all the ions. We include many configurations together with inner-subshell excited ones such as $4 \mathrm{p}^{5} 4 \mathrm{~d}^{n}$. Using the 


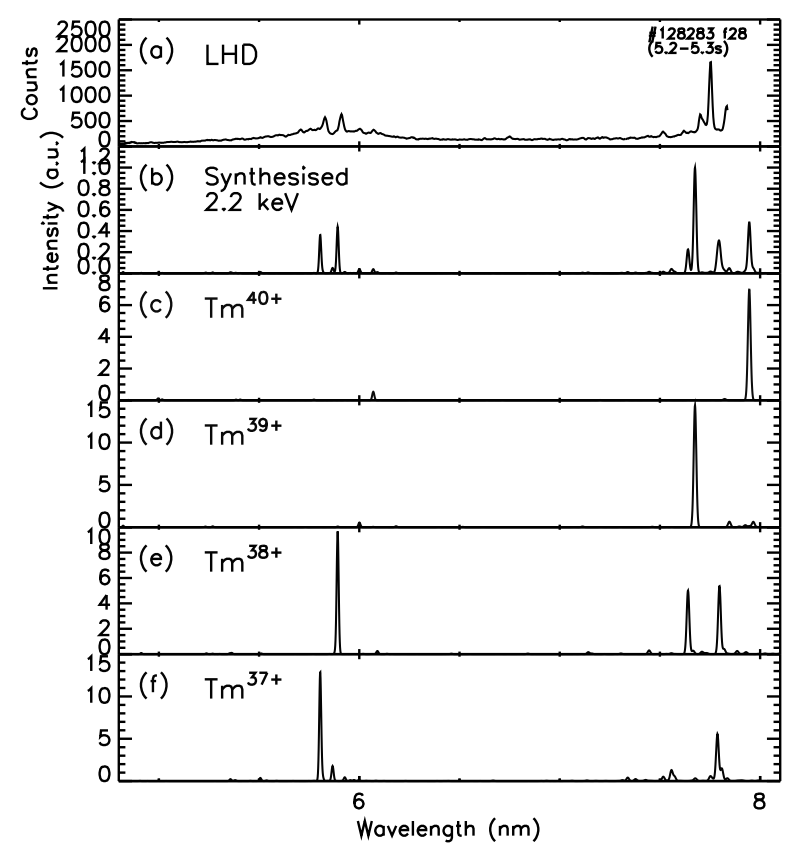

Figure 10. Comparison between the measured and modelled spectra from Tm ions at a temperature of $2.2 \mathrm{keV}$ : (a) spectrum measured in LHD, (b) synthesised spectrum with assuming the abundance ratio of 0.625:1.0:1.521:1.5 for $\mathrm{Tm}^{37+}: \mathrm{Tm}^{38+}: \mathrm{Tm}^{39+}: \mathrm{Tm}^{40+}$ and (c)-(f) calculated spectra for each ion.

model, we have successfully evaluated the fractional abundances of tungsten ions $\mathrm{W}^{21+}$ $\mathrm{W}^{33+}$ at different electron temperatures based on the measured $\Delta n \neq 0$ UTA spectra in the 1.5-3.5 nm region as described in [18]. However, the $\Delta n=0$ UTA spectra in the $4-$ $7 \mathrm{~nm}$ region are not well reproduced with this model. An earlier study using other codes and atomic data suggests that dielectronic recombination processes might contribute to the $\Delta n=0$ UTA feature [36]. Further improvement of the model is required to examine their contributions.

We are now trying to extend the model to lanthanide elements. For example, a comparison between the measured and modelled spectra from Tm ions in a high temperature condition is shown in figure 10. The abundance ratios for $\mathrm{Tm}^{37+}-\mathrm{Tm}^{40+}$ have been tuned so as to reproduce the measured line intensity ratios. The calculated wavelengths of the lines around $5.9 \mathrm{~nm}$ agree with the measurements within $\pm 0.02 \mathrm{~nm}$, while those around $7.5-8 \mathrm{~nm}$ are shifted by $0.06-0.08 \mathrm{~nm}$ to shorter wavelength. Nevertheless, the measured spectral feature was qualitatively well reproduced by the synthesised spectrum from $\mathrm{Cu}-, \mathrm{Zn}-$, Ga- and Ge-like ions calculated for the measured electron density and temperature $(2.2 \mathrm{keV})$. Therefore, the present results possibly would lead to further new experimental identifications of isolated spectral lines. We will try to further improve the models so as to reproduce the UTA features of the lanthanide elements in low temperature conditions. 


\section{Summary}

We have so far carried out studies on EUV spectroscopy of a number of heavy ions in the 5th and 6th period elements using LHD plasmas. Spectra from highly charged ions having $N$ shell outermost electrons appear as discrete or quasi-continuum UTA features depending on the electron temperature. The characteristic behaviour of the $n=4-4$ UTA structure due to the effect of $n=4$ electron correlation have been found consistent with the calculations. Some of the isolated spectral lines of $\mathrm{Nd}$, Tb, Ho and $\mathrm{Tm}$ ions with relatively simple configurations have been newly found in the LHD. The CR model developed for the measured EUV spectra of tungsten ions is being extended to the other elements. The present study demonstrates that the LHD is a good complementary platform for studying EUV spectra from highly charged heavy ions because of its low opacity condition and availability of high quality diagnostic tools.

\section{Acknowledgments}

The authors acknowledge the LHD experiment group for their assistance. This work was carried out with the support and under the auspices of the NIFS collaboration research program (NIFS15KLPF038, NIFS16KLPH026). This work was supported also by JSPS KAKENHI Grant Numbers $15 \mathrm{H} 03759$ and $15 \mathrm{H} 04234$.

\section{References}

[1] Finkenthal M, Huang L K, Lippmann S, Moos H W, Mandelbaum P, Schwob J L, Klapisch M and TEXT Group 1988 Phys. Lett. A 127255

[2] Hinnov E, Suckewer S, Cohen S and Sato K 1982 Phys. Rev. A 252293

[3] May M J, Finkenthal M, Moos H W, Fournier K B, Goldstein W H, Mattioli M, Pacella D, Mazzitelli G, Leigheb M and Gabellieri L 2001 Phys. Rev. E 64036406

[4] Kaneko O et al 2013 Nucl. Fusion 53104015

[5] Narihara K, Yamada I, Hayashi H and Yamauchi K 2001 Rev. Sci. Instrum. 721122

[6] Yamada I et al 2010 Rev. Sci. Instrum. 81 10D522

[7] Sudo S and Tamura N 2012 Rev. Sci. Instrum. 83023503

[8] Nozato H, Morita S, Goto M, Ejiri A and Takase Y 2003 Rev. Sci. Instrum. 742032

[9] Schwob J L, Wouters A W, Suckewer S and Finkenthal M 1987 Rev. Sci. Instrum. 581601

[10] Chowdhuri M B, Morita S, Goto M 2008 Appl. Opt. 47135

[11] Chowdhuri M B, Morita S, Goto M, Nishimura H, Nagai K and Fujioka S 2007 Plasma Fusion Res. 2 S1060

[12] Yamamoto N, Kato T, Funaba H, Sato K, Tamura N, Sudo S, Beiersdorfer P and Lepson J K 2008 Astrophys. J. 689646

[13] Suzuki C et al 2010 J. Phys. B: At. Mol. Opt. Phys. 43074027

[14] Murakami I et al 2014 Plasma Fusion Res. 91401056

[15] Suzuki C et al 2014 Phys. Scr. 89114009

[16] Harte C S et al 2010 J. Phys. B: At. Mol. Opt. Phys. 43205004

[17] Suzuki C et al 2011 J. Phys. B: At. Mol. Opt. Phys. 44175004

[18] Murakami I, Sakaue H A, Suzuki C, Kato D, Goto M, Tamura N, Sudo S, Morita S and LHD Experiment Group 2015 Nucl. Fusion 55093016

[19] Nishihara K et al 2008 Phys. Plasmas 15056708 
[20] O'Sullivan G and Faulkner R 1994 Opt. Eng. 333978

[21] Churilov S S, Kildiyarova R R, Ryabtsev A N and Sadovsky S V 2009 Phys. Scr. 80045303

[22] Otsuka T, Kilbane D, White J, Higashiguchi T, Yugami N, Yatagai T, Jiang W, Endo A, Dunne P and O'Sullivan G 2010 Appl. Phys. Lett. 97111503

[23] Higashiguchi T, Otsuka T, Yugami N, Jiang W, Endo A, Li B and O'Sullivan G 2012 Appl. Phys. Lett. 100014103

[24] Kato T et al 2008 J. Phys. B: At. Mol. Opt. Phys. 41035703

[25] Suzuki C, Koike F, Murakami I, Tamura N and Sudo S 2012 J. Phys. B: At. Mol. Opt. Phys. 45 135002

[26] Suzuki C, Koike F, Murakami I, Tamura N and Sudo S 2015 J. Phys. B: At. Mol. Opt. Phys. 48 144012

[27] Ohashi H et al 2015 J. Phys. B: At. Mol. Opt. Phys. 48144011

[28] Bauche J, Bauche-Arnoult C and Klapisch M 1988 Phys. Scr. 37659

[29] Gu M F 2008 Can. J. Phys. 86675

[30] Harte C S, Higashiguchi T, Otsuka T, D'Arcy R, Kilbane D and O'Sullivan G 2012 J. Phys. B: At. Mol. Opt. Phys. 45205002

[31] Nagaoka K et al 2015 Nucl. Fusion 55113020

[32] Koike F and Fritzsche S 2007 Rad. Phys. Chem. 76404

[33] Koike F et al 2013 AIP Conf. Proc. 1545202

[34] Ohashi H et al 2014 Appl. Phys. Lett. 104234107

[35] Bar-Shalom A, Klapisch K and Oreg J 2011 J. Quant. Spectrosc. Radiat. Transfer 71169

[36] Pütterich T, Jonauskas V, Neu R, Dux R and ASDEX Upgrade Team 2013 AIP Conf. Proc. 1545 132 